\title{
The unusual structure detection in Extensive air shower events at Horizon-8T cosmic rays detector system
}

\author{
Rashid Beisembaev, Elena Beisembaeva, Oleg Dalkarov, Vladimir Ryabov, Sergei \\ Shaulov, Marina Vildanova, Valeriy Zhukov \\ P. N. Lebedev Physical Institute of the Russian Academy of Sciences \\ 53 Leninskiy avenue, Moscow, Russia
}

Dmitriy Beznosko, Alexander lakovlev

Bard Early College New Orleans

New Orleans, USA

\author{
Ayan Batyrkhanov ${ }^{1}$, Kanat Baigarin, Tileubek Uakhitov, Alikhan Yeltokov \\ Nazarbayev University \\ 53 Kabanbay Batyr ave., Astana, Kazakhstan \\ E-mail: ayan.batyrkhanovenu.edu.kz
}

\section{Turlan Sadykov}

LLP "Institute of Physics and Technology"

Almaty, Kazakhstan

\begin{abstract}
Horizon-8T is a detector system aimed to study Extensive Air Showers (EAS) temporal structure in the energy range of the primary above $\sim 10^{16} \mathrm{eV}$. It is constructed at approximately 3340 meters above the sea level at the Tien Shan high-altitude Science Station, part of Lebedev Physical Institute of the Russian Academy of Sciences. Detector system consists of eight charged particle detection points separated by the distance up to one kilometer.

During Physics Run 1 from October 2016 to April 2017, about 8000 total events were detected, a sizable number of which exhibit the unusual spatial and temporal structure of pulses with several maxima (or modes). The separation of the maxima can be from few tens of ns to several hundred ns. The Run 1 dataset suggests that separation between maxima increases with distance from EAS core, which cannot be obtained from simulations, and seem to occur only in events with energy above $\sim 10^{17} \mathrm{eV}$. The overview of Horizon-8T detector system and the details of the unusual events data will be presented.
\end{abstract}

The 39th International Conference on High Energy Physics (ICHEP2018)

4-11 July, 2018

Seoul, Korea 


\section{Introduction}

The Horizon-8T (HT) detector is an upgraded version of the Horizon-T detector system [1] with 8 working detection points and additional two detectors under constructions. HT was constructed at the Tien Shan High-altitude Science Station (TSHSS), Almaty, Kazakhstan, and is located at $\sim 3340$ meters above the sea level. Detector system is aimed to study spatial and temporal distribution of the EAS charged particles. Aerial view of the system is illustrated on Figure 1. Detector includes near periphery detection points (1, 4 to 7 points in Figure 1) and far periphery (points 2, 3, 8 to 10 in Figure 1). The spatial separation between two farthest detectors is approximately 900 meters. With the upgrade of two extra detection points separation will become $\sim 1.3 \mathrm{~km}$. In addition to the main detector points optical detector subsystem is used to detect Vavilov-Cherenkov light. Vavilov-Cherenkov detector is located at the Center (1st detection point in Figure 1) and is associated with Hamamatsu [2] H6527 PMT.

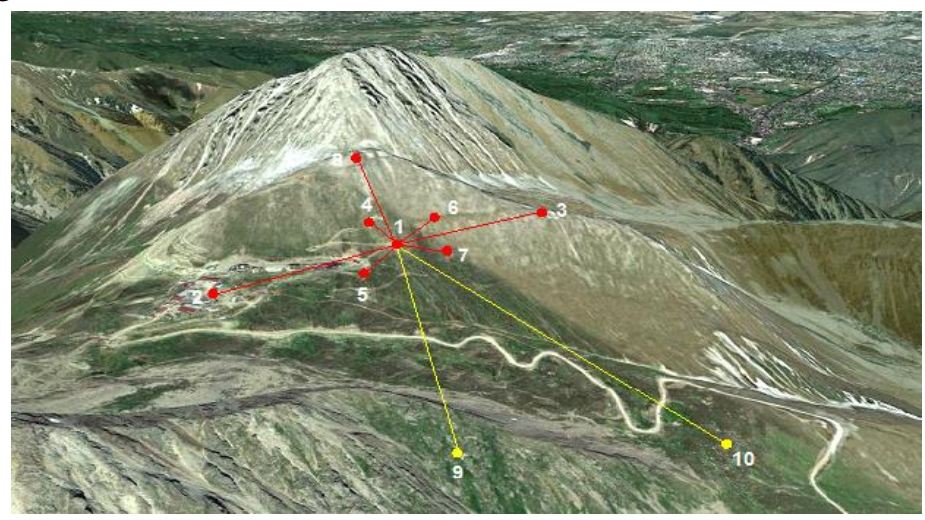

Figure 1. Aerial view of HT detector system with 8 working detectors (red color) and additional detectors (yellow color)

Hardware trigger for the system is the coincidence between detection points 5 and 6 with the threshold of about 5 minimally ionizing particles (MIP) for each (background cosmic rays). Each detection point consists of $1 \mathrm{~m}^{2}$ and $5 \mathrm{~cm}$ thickness plastic polystyrene-based scintillator detectors [3] and fast Hamamatsu R7723 or H6527 PMT detectors. All near periphery detectors are additionally equipped with glass detectors with R7723 PMT of rise time response $\sim 2.3 \mathrm{~ns}$. Plastic scintillator contribution is $\sim 5 \mathrm{~ns}$ that gives the total time response of 7-8 ns. Data acquisition (DAQ) system is based on 3 CAEN [4] desktop analog to digital converter (ADC) modules with $500 \mathrm{MHz}$ digitization rate. Two physics I (2016-2017) and physics II (2017-2018) runs have been collected.

\section{Standard EAS events}

Standard EAS [5] disks were defined by EAS events simulated by CORSIKA simulation package [6]. These events were used to identify properties of the standard disks. Simulation results showed that the particle density drops rapidly with the distance from the axis. Temporal width of the disk was defined as the time between $10 \%$ and $90 \%$ of the particles detected. Disk arrival time is the time of the $50 \%$ of the particles arrived to the detector. Results of the arrival time and disk width dependence on the distance from the disk axis are illustrated on Figure 2. Time is measured in ns. Arrival time and time width were fitted with quadratic and linear functions. Far from the axis width of the disk is measured inaccurately and it deviates from the linear plot because of the low particle density. 

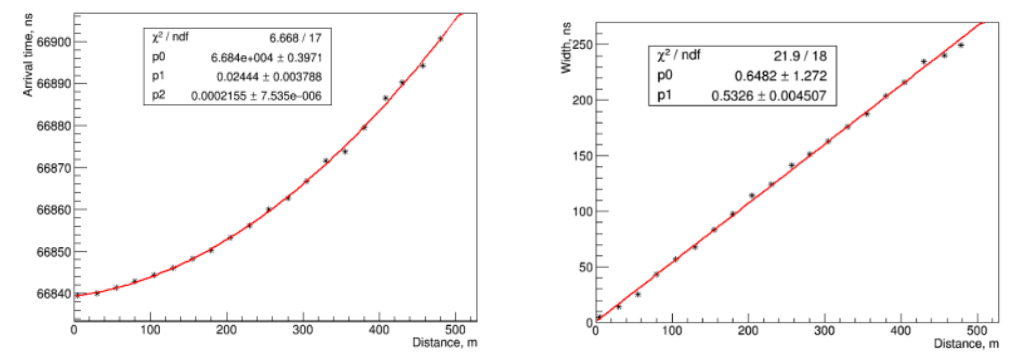

Figure 2. Simulation results of disk arrival time dependence on distance from the axis (left) and disk width dependence on the distance from the disk axis (right)

Both particle density characteristic and disk temporal width can be used for event reconstruction. Disk arrival time provides more opportunities to reconstruction. Example of the standard event with energy of the primary of $\sim 2 \times 10^{16} \mathrm{eV}$ is presented on the Figure 3 .

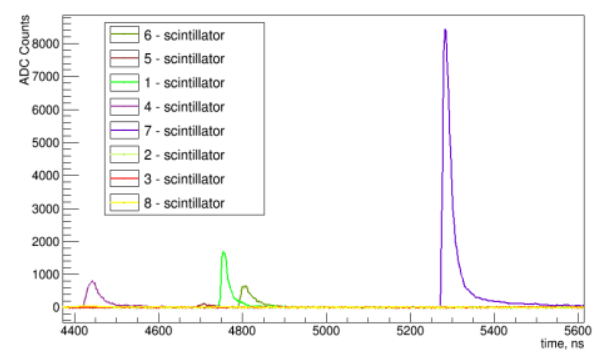

Figure 3. Standard EAS event detected on H8T detector system

\section{Unusual EAS events}

Some events detected during Physics Run 1 and Physics Run 2, however, have behavior different from the standard events described above. The main characteristic of these 'unusual' events are peaks consisting of several smaller peaks. Event presented on Figure 4 (detected on February 24,2018 ) clearly shows the trend. Axis of this event was detected near the detection point 7 closer to point 6 . This event is an example of typical unusual event that follows multipeak behavior. Distance between the peaks increasing with the distance to the axis, which is the main characteristic of such unusual events. This property is common for all other unusual events detected at H8T.
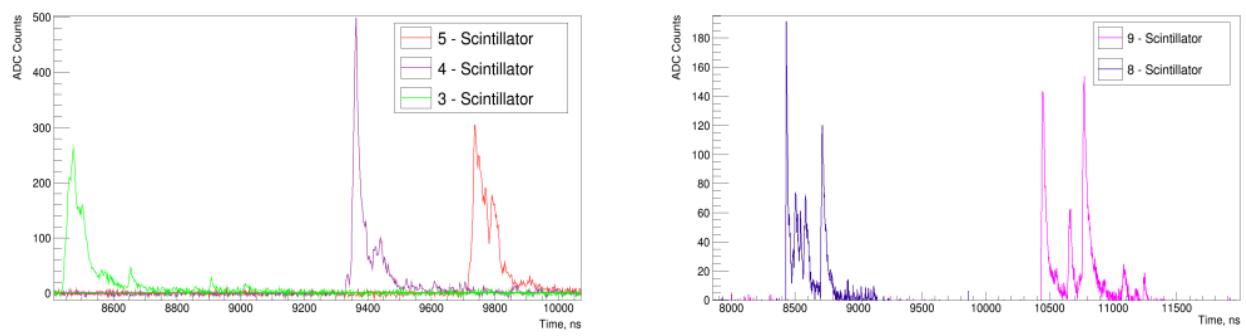

Figure 4. Unusual EAS event with axis at 7. Detection points closer to the axis (left, points 3,4,5) and far from the center (right, 8,9)

\section{Unusual events construction using standard events}

Hypothesis that unusual events described above can be simulated by combination of two standard EAS disks was checked. EAS disks were simulated using CORSIKA software. Two combination approaches were applied. Disks that are detected on the detection level with time delay between them and at the same distance from their axes give the combination results shown on Figure 5. Time delay between the centers of the disks is $100 \mathrm{~ns}$. Energy of the primary for each EAS event is $10^{17} \mathrm{eV}$. 

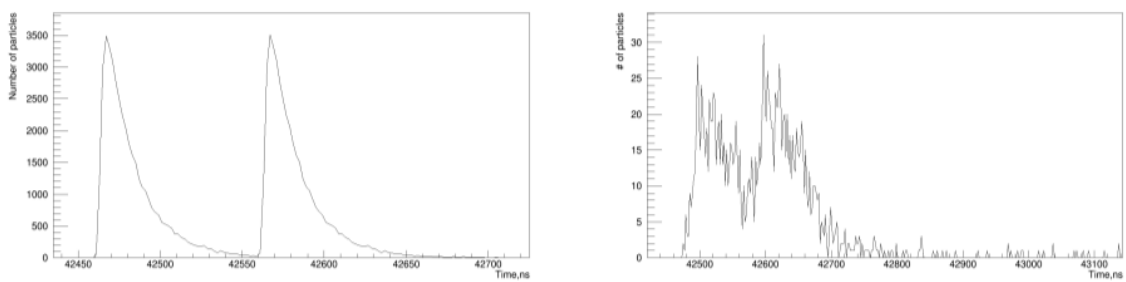

Figure 5. Disks combination with time delay at $50 \mathrm{~m}$ (left) and $170 \mathrm{~m}$ (right) from their axes

As can be seen from the Figure 5 width of the disks increases with the distance (matches with the simulation results on Figure 2, right). It leads to the detection of two easily differentiated high-amplitude pulses close to the center of the disks and hardly distinguishable multipeak event with low amplitude far from the axis. This behavior does not fit the structure of the unusual events.

Another approach to simulate the unusual events was to combine two disks at different distances from their axes without time delay. Combinations of such events are shown on Figure 6. No time delay between disks was set. Energy of the primary for each event is $10^{16} \mathrm{eV}$ for the close to the center disks (Figure 6, left) and $10^{17} \mathrm{eV}$ for the far from the center events (Figure 6, right). As the particle density decreases with the distance to the axis of the disk, contribution of the second disk at $90 \mathrm{~m}$ is not visible compared to the event at $30 \mathrm{~m}$ from the axis (Figure 6, left). Even far from the center at 130 and 170 meters (Figure 6, right) particle density drop leads to the non-distinguishable peaks, thus, giving no multipeak behavior.
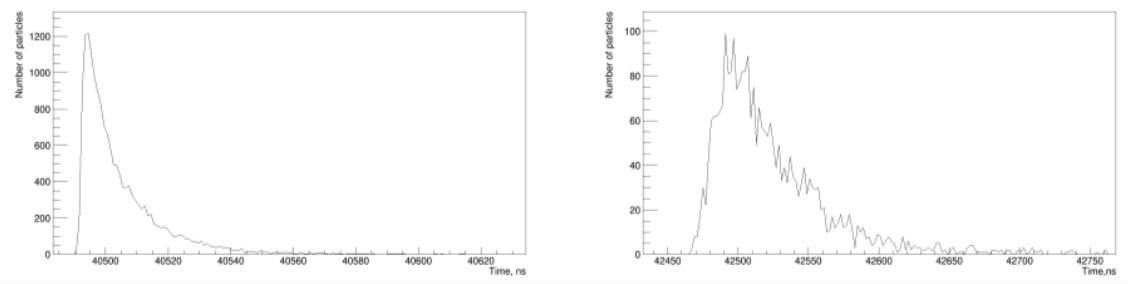

Figure 6. Disks combination with spatial separation at 30 and $90 \mathrm{~m}$ (left) and 130 and $170 \mathrm{~m}$ (right) from their axes

\section{Conclusion}

Horizon-8T detector system is designed and constructed for unusual event detection. During two Physics Runs such events were detected and their behaviour was identified. Simple combinations of the simulated disks do not give the same structure. Further data collection and analysis is continued. Far periphery detectors with larger area are under upgrade.

\section{Acknowledgments}

This work was funded in part by MES RK state-targeted program BR95236464.

\section{References}

[1] R U Beisembaev et al., arXiv:1605.05179 (2016)

[2] Hamamatsu Photonics, 314-5 Shimokanzo, Toyookavillage, Iwatagun, Shizuoka-ken, 438-0193 Japan, http://www.hamamatsu.com

[3] Dmitriy Beznosko et al., EPJWeb of Conferences 145, 19016 (2017)

[4] CAEN S.p.A. Via della Vetraia, 11, 55049 Viareggio Lucca, Italy http://www.caen.it

[5] Dmitriy Beznosko et al., EPJWeb of Conferences 145, 14001 (2017)

[6] D. Heck et al., Forschungszentrum Karlsruhe Report FZKA 6019 (1998) 